\title{
Influence of CDK5 Regulatory Subunit-Associated Protein I-Like I Expression on the Survival of Patients with Non-Metastatic Nasopharyngeal Carcinoma
}

\author{
Zhanzhan Li (iD) \\ Yajie Zhao ${ }^{2}$ \\ 'Department of Oncology, Xiangya \\ Hospital, Central South University, \\ Changsha, Hunan Province, 410008, \\ People's Republic of China; ${ }^{2}$ Department \\ of Nuclear Medicine, Xiangya Hospital, \\ Central South University, Changsha, \\ Hunan Province, 410008, People's \\ Republic of China
}

\begin{abstract}
Background: The aim of this study was to investigate the influence of CDK5 regulatory subunit-associated protein 1-like 1 (CDKAL1) expression on the survival of nasopharyngeal carcinoma (NPC) patients.

Methods: A total of 140 non-metastatic NPC patients were retrospectively analyzed. The expression of CDKAL1 was detected by immunohistochemistry.

Results: The CDKAL1-negative group exhibited better overall survival (OS) according to Kaplan-Meier curve analysis $(p=0.006)$, with 5-year OS rates for the CDKAL1-negative and -positive groups of $89.7 \%$ and $70.2 \%$, respectively. The CDKAL1-negative group also exhibited a tendency toward a better progression-free survival (PFS) rate. Multivariate analysis showed that CDKAL1 expression was independently associated with both OS $(p=0.002)$ and PFS $(p=0.043)$.
\end{abstract}

Conclusion: CDKAL1 expression is an independent negative predictor of patient survival in non-metastatic NPC.

Keywords: nasopharyngeal carcinoma, CDKAL1, overall survival, progression-free survival

\section{Introduction}

With the application of modern treatment modalities, the prognosis of nasopharyngeal carcinoma (NPC) has significantly improved. ${ }^{1,2}$ For non-metastatic NPC, the 5-year overall survival (OS) has been reported to be between $62 \%$ and $90 \%{ }^{3-6}$ The tumor-node-metastasis (TNM) staging system is the most widely used system for predicting the survival of NPC patients; however, due to the intrinsic heterogeneity of tumor cells, and because intratumor microenvironments are not considered in the TNM staging system, personalized biomarkers for the pathogenesis or progression of NPC are needed to predict patient survival more accurately.

CDK5 regulatory subunit-associated protein 1-like 1 (CDKAL1) is a proteincoding gene involved in post-transcriptional RNA modification. ${ }^{7}$ Genome-wide association studies have shown that CDKAL1 is associated with susceptibility to type 2 diabetes. ${ }^{8,9}$ Other studies have indicated that CDKAL1 may play a role in the carcinogenesis and prognosis of several types of cancer, including both breast and endometrial cancers; ${ }^{10,11}$ however, the role of CDKAL1 in NPC has not yet been
Correspondence: Yajie Zhao Department of Nuclear Medicine, Xiangya Hospital, Central South University, No. 87, Xiangya Road, Changsha, Hunan Province, 410008,

People's Republic of China

Tel +86-73I-89753769

Emailwzyj7I77@I26.com 
investigated. Therefore, we sought to examine the influence of CDKAL1 expression on the survival of patients with non-metastatic NPC.

\section{Materials and Methods}

\section{Patient Selection}

A total of 140 NPC patients were selected according to the following criteria: (1) pathologically confirmed nonmetastatic NPC patients [according to the American Joint Committee on Cancer (AJCC), staging system (7th edition)]; (2) received definitive radiotherapy with or without other treatments at Xiangya Hospital, Central South University between August 2008 and January 2012; and (3) availability of pathology specimens for immunohistochemistry. The study was approved by the Research and Ethics Committee of Xiangya Hospital. Written informed consent was obtained from all subjects. This study was conducted in accordance with the Declaration of Helsinki.

\section{Treatments}

All patients were treated with intensity-modulated radiation therapy (IMRT). The target volumes included the gross tumor volume of the nasopharynx (GTVnx), the gross tumor volume of lymph nodes (GTVnd), clinical target volume 1 (CTV1), and clinical target volume 2 (CTV2). CTV1 and CTV2 were defined as the high- and low-risk volume, respectively. Areas of 3-5 mm around the GTVnx, GTVnd, CTV1, and CTV2 were included in the planning target volumes (PGTVnx, PGTVnd, PTV1, and PTV2, respectively). The doses to the PGTVnx, PGTVnd, PTV1, and PTV2 were 66-73.92 Gy (33 fractions), 59.6 to $72-$ Gy (33 fractions), 50.4-66 Gy (33 fractions), and 50.4-61.05 Gy (33 fractions), respectively. The dose limits for the organs at risk (OARs), and the PTVs, were based on protocol 0225 of the Radiation Therapy Oncology Group (RTOG). ${ }^{12}$

Neoadjuvant chemotherapy was administered when the waiting time for radiotherapy was too long or the tumor was too large. Concurrent chemotherapy was administered in patients with cancer stage II-IV. Adjuvant chemotherapy was administered in $\mathrm{N} 2 / \mathrm{N} 3$ patients, and in patients with residual tumor at the end of radiotherapy. All chemotherapy regimens were platinum-based. Patients who were unwilling to receive, or could not tolerate, chemotherapy did not undergo chemotherapy. In addition, some patients received targeted therapy to inhibit epidermal growth factor receptor (EGFR), including nimotuzumab and cetuximab, concurrent with radiotherapy. Nimotuzumab was administered at a dose of 100 $200 \mathrm{mg} /$ week. Meanwhile, cetuximab was initially administered at a dose of $400 \mathrm{mg} / \mathrm{m}^{2}$, followed by $250 \mathrm{mg} / \mathrm{m}^{2} /$ week.

\section{Immunohistochemistry of CDKALI}

Formalin-fixed, paraffin-embedded slides were processed by deparaffinization, rehydration, antigen retrieval, and blockade of endogenous peroxidase. Subsequently, the specimens were incubated overnight at $4^{\circ} \mathrm{C}$ in the presence of anti-CDKAL1 antibody (Abcam, Cambridge, UK) at a dilution of 1:200. Immunostaining was performed with a ChemMate kit (Dako, Glostrup, Denmark), with 3.3-diaminobenzidine used as the chromogenic substrate. A control group was included to ensure the quality of immunostaining, in which the primary antibody was replaced with nonimmune isotypic antibodies.

The staining results were independently assessed by two pathologists blinded to the patient data. The data were analyzed using the immunoreactive score (IRS) system, which took into consideration the extent and intensity of CDKAL1 staining. The scores were as follows: $0=<$ $10 \%$ of cells weakly stained, $1=11-30 \%$ of cells weakly stained, $2=>30 \%$ of cells weakly stained or $<30 \%$ of cells moderately stained, $3=30-60 \%$ of cells moderately stained, and $4=>60 \%$ of cells moderately or strongly stained. ${ }^{13}$ The final score was defined as the mean score of five independent fields. In the current study, cases with a final score of $0-1$ point were defined as CDKAL1negative, while those with a final score of $2-4$ points were defined as CDKAL1-positive. A typical case with negative CDKAL1 expression, and a typical case with positive CDKAL1 expression, are shown in Figure 1.

\section{Univariate and Multivariate Analyses of OS and PFS}

To further confirm the influence of CDKAL1 expression on patient survival, univariate and multivariate analyses were performed to identify factors independently associated with OS and PFS. In addition to CDKAL1 expression, factors that have been reported to be associated with survival outcomes of NPC, such as age ( $<45$ vs $\geq 45$ years), gender, clinical stage, chemotherapy, anti-EGFR targeted therapy, and the prescription dose to the primary tumor, were included in the univariate analysis. ${ }^{14-18}$ All variables with an $\alpha$ value $<0.2$ in the univariate analysis, as well as those regarded as 

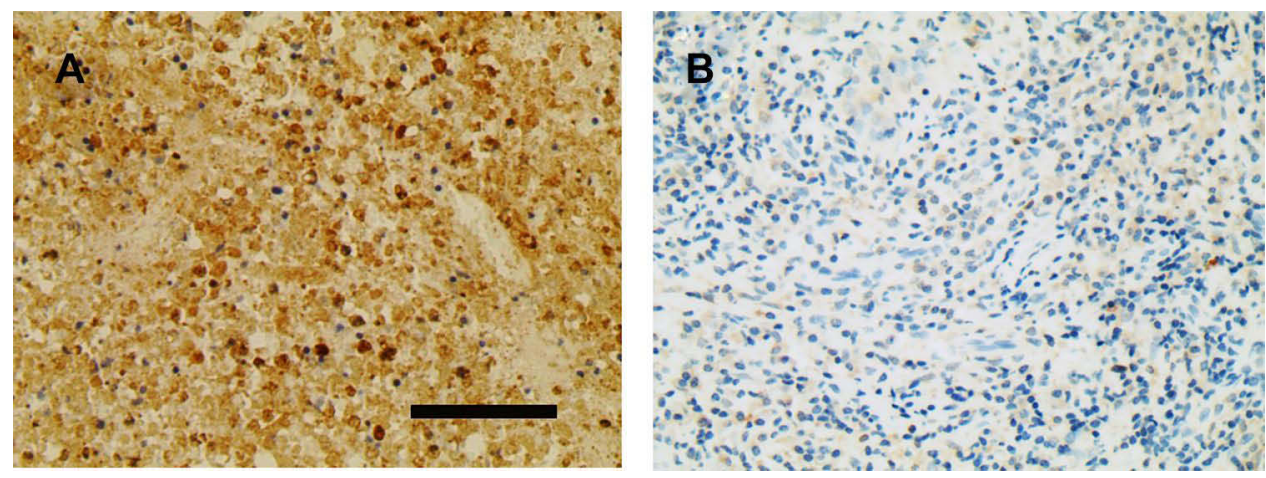

Figure I A typical case with positive CDKALI expression $(\mathbf{A})$ and a typical case with negative CDKALI expression $(\mathbf{B})$. Scale bar $=90 \mu \mathrm{m} ;$ magnification $\times 400$.

important for patient survival in clinical practice (such as chemotherapy), were included in the multivariate analysis.

\section{Follow-Up and Statistical Analysis}

The OS time was defined as the interval between initial diagnosis and death from any cause, or the last follow-up for surviving patients. PFS time was defined as the interval between initial diagnosis and disease progression or the last follow-up. All of the statistical analyses were performed using SPSS software (version 25.0; IBM Corp., Armonk, NY, USA). Comparisons of clinical characteristics between the CDKAL1-negative and -positive groups were performed with the chi-squared test. The Kaplan-Meier method was used to plot survival curves and calculate survival rates. The survival differences between the CDKAL1-negative and positive groups were analyzed with the Log rank test. Univariate and multivariate analyses of prognostic factors for OS and PFS were conducted with the Cox proportional hazards model.

\section{Results}

\section{Patient Characteristics}

The characteristics of the CDKAL1-negative and -positive groups are listed in Table 1. No significant group differences were observed in age ( $<45$ vs $\geq 45$ years), gender, rates of chemotherapy and anti-EGFR targeted therapy, or the dose to the PGTVnx ( $<70.4$ vs $\geq 70.4$ Gy). However, significant differences in both clinical stage $(p=0.025)$ and T stage $(p=0.007)$ were observed between the two groups. The IHC score ranged from 0 to 4 points, with a mean value of 1.88 . The mean IHC score was significantly higher in the CDKAL1-positive than -negative group $(2.20 \pm 1.12$ vs $1.36 \pm 1.13, p<0.001)$.

\section{Influence of CDKALI Expression on OS}

The median follow-up time was 65 months. Figure 2A shows the Kaplan-Meier OS curve for all patients. The 3and 5-year OS rates for all patients were $85.7 \%$ and $77.6 \%$, respectively. As shown in Figure 3A, there was a significant difference in OS between the CDKAL1-negative and positive groups $(p=0.006)$, for which the 3 -year OS rates were $92.5 \%$ and $81.6 \%$, and the 5 -year OS rates $89.7 \%$ and 70.2\%, respectively. As shown in Table 2, CDKAL1 expression $[p=0.002$; hazard ratio $(\mathrm{HR})=4.662,95 \%$ confidence interval (CI): 1.748-12.221] was independently associated with OS.

\section{Influence of CDKALI Expression on PFS}

Figure 2B shows the Kaplan-Meier curve of PFS for all patients. The 3- and 5-year PFS rates for all patients were $74.8 \%$ and $65.2 \%$, respectively. As shown in Figure 3B, the CDKAL1-negative group displayed a tendency toward a better PFS rate relative to the CDKAL1-positive group, although the difference was not statistically significant $(p=$ 0.265 ). The 3 -year PFS rates were $81.1 \%$ and $71 \%$, and the 5 -year PFS rates were $70.8 \%$ and $61.9 \%$, for the CDKAL1negative and -positive groups, respectively. As shown in Table 3, CDKAL1 expression ( $p=0.043$; HR $=1.903,95 \%$ CI: 1.020-3.551) was independently associated with PFS.

\section{Discussion}

CDKAL1 is a known susceptibility gene for type 2 diabetes. Recent studies have indicated that CDKAL1 is also involved in the carcinogenesis and prognosis of several types of cancer, ${ }^{10,11}$ although its role in NPC is unclear. To our knowledge, the current study is the first to investigate the influence of CDKAL1 expression on the survival of patients with non-metastatic NPC. 
Table I Comparison of Clinical Characteristics Between the CDKALI-Negative Group and the CDKALI-Positive Group

\begin{tabular}{|c|c|c|c|}
\hline & $\begin{array}{c}\text { CDKALI- } \\
\text { Negative } \\
\text { Group }(n=53)\end{array}$ & $\begin{array}{c}\text { CDKALI- } \\
\text { Positive Group } \\
(n=87)\end{array}$ & p-value \\
\hline Age & $47.9 \pm 12.1$ & $47.3 \pm 11.1$ & 0.765 \\
\hline$<45 y$ & 23 & 35 & 0.712 \\
\hline$\geq 45 y$ & 30 & 52 & \\
\hline Gender & & & 0.409 \\
\hline Male & 40 & 60 & \\
\hline Female & 13 & 27 & \\
\hline Clinical stage & & & 0.025 \\
\hline II & 2 & 15 & \\
\hline III & 14 & 28 & \\
\hline IV & 37 & 44 & \\
\hline $\mathrm{T}$ & & & \\
\hline TI-T2 & 3 & 20 & 0.007 \\
\hline T3-T4 & 50 & 67 & \\
\hline $\mathrm{N}$ & & & 0.279 \\
\hline No/NI & 18 & 38 & \\
\hline N2/N3 & 35 & 49 & \\
\hline Chemotherapy & & & 0.450 \\
\hline No & 4 & 10 & \\
\hline Yes & 49 & 77 & \\
\hline $\begin{array}{l}\text { Anti-EGFR } \\
\text { targeted therapy }\end{array}$ & & & 0.057 \\
\hline No & 43 & 80 & \\
\hline Yes & 10 & 7 & \\
\hline $\begin{array}{l}\text { Prescription } \\
\text { dose of PGTVnx }\end{array}$ & & & 1.000 \\
\hline$<70.4$ Gy & 4 & 7 & \\
\hline$\geq 70.4$ Gy & 49 & 80 & \\
\hline IHC score & $1.36 \pm 1.13$ & $2.20 \pm 1.12$ & 0.000 \\
\hline
\end{tabular}

Abbreviations: PGTVnx, the planning target volume of the gross tumor volume of nasopharynx pharynx; IHC, Immunohistochemistry.

Our results showed that CDKAL1 expression was negatively associated with the OS of non-metastatic NPC patients, as evidenced by both the Kaplan-Meier curves and multivariate analysis. With respect to PFS, although no statistically significant difference between the CDKAL1-negative and -positive groups was observed based on the Kaplan-Meier curves, the CDKAL1negative group showed a tendency toward improved PFS. Furthermore, multivariate analysis showed that CDKAL1 expression was independently and negatively associated with PFS ( $p=0.042$; HR $=1.910)$. One possible explanation for the non-significant difference in the Kaplan-Meier PFS curves may have been the imbalanced distribution
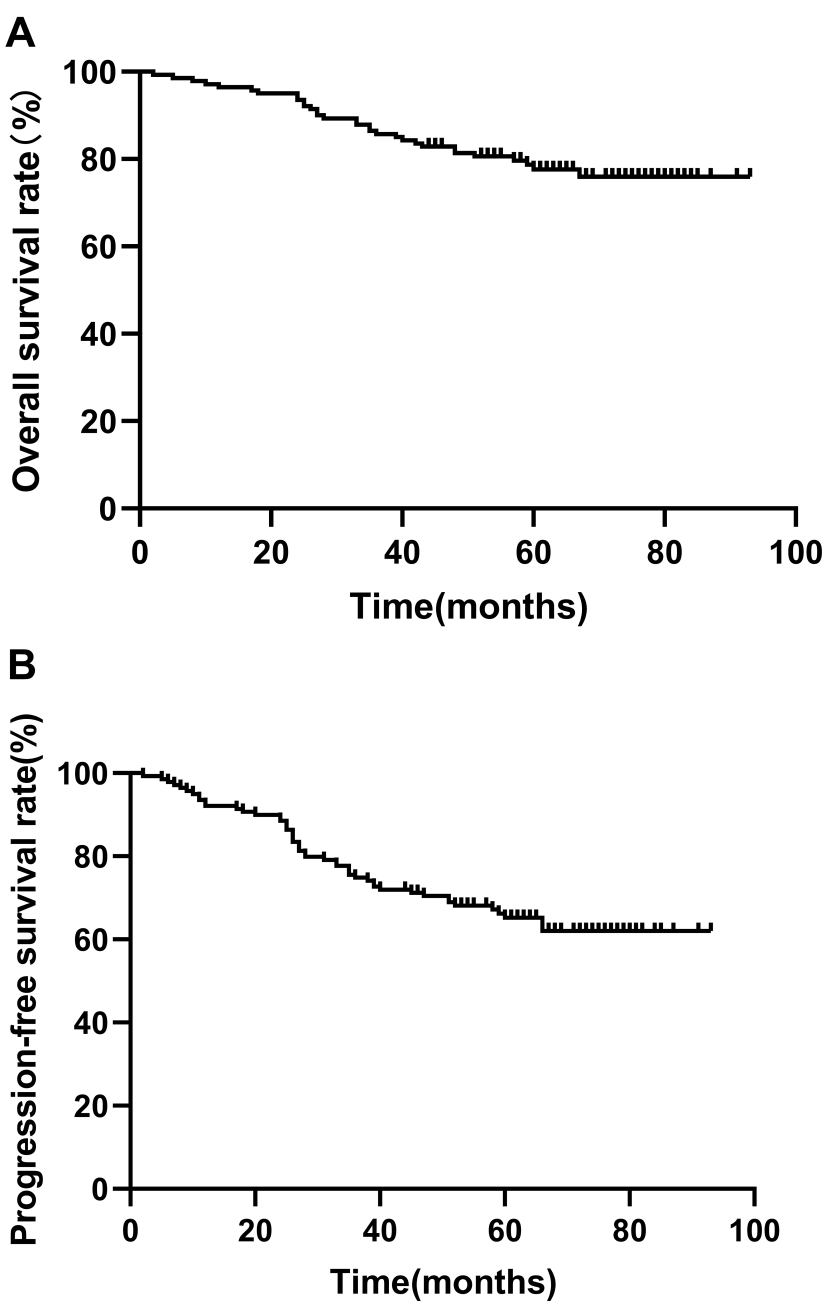

Figure 2 Kaplan-Meier curves of OS (A) and PFS (B) of all patients.

clinical stages between the two groups ( $p=0.025)$, which can have a significant impact on PFS. ${ }^{16,19}$ Therefore, CDKAL1 expression is considered a negative predictor of both OS and PFS in non-metastatic NPC.

Several studies have indicated that CDKAL1 is negatively associated with disease prognosis in cancer patients. Data from the human protein atlas suggested that the expression of CDKAL1 predicts poor OS in endometrial cancer $(p<0.001)$, with high expression of CDKAL1 possibly acting as an early event in tumor progression in bladder cancer. ${ }^{20}$ In addition, a study by Parada et al suggested that a single-nucleotide polymorphism (SNP) of CDKAL1 (rs981042) was associated with an increased risk of death in breast cancer patients. ${ }^{11}$ Furthermore, CDKAL1 has also been reported to be involved in tumor carcinogenesis in several types of cancer, including endometrial cancer, colorectal cancer, and breast cancer. ${ }^{21}$ The data presented here are broadly consistent with these 


\section{A}
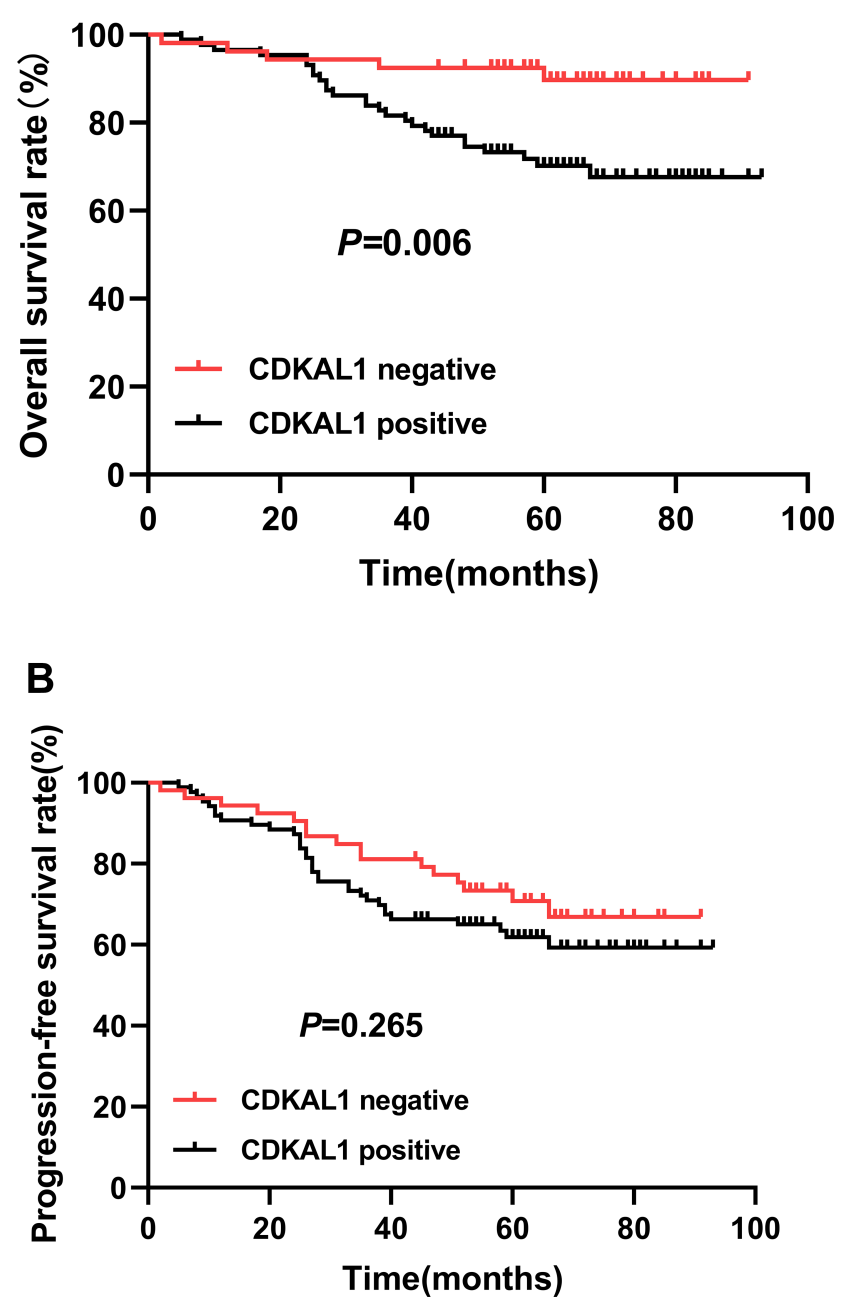

Figure 3 Kaplan-Meier curves of OS (A) and PFS (B) of the CDKALI-negative group (red) and the CDKALI-positive group (black).

findings, suggesting a strong negative association between CDKAL1 expression and carcinogenesis.

It is worth mentioning that the mechanism underlying the association between CDKAL1 and cancer prognosis or carcinogenesis remains unknown, although the role of CDKAL1 in the prognosis of NPC patients can be explained by the following mechanism. The protein encoded by this gene is a member of the methylthiotransferase family. CDKAL1 protein is highly homologous to cyclin-dependent kinase 5 regulatory subunit associated protein 1 (CDK5RAP1), which acts as an inhibitor of CDK5 by binding to CDK5-activator p35. CDK5 has been shown to be involved in the epithelial-tomesenchymal transition in various types of cancers, including head and neck squamous cell carcinoma. ${ }^{22-24}$ A previous study indicated that ARNTL suppresses NPC cell proliferation and enhances sensitivity to cisplatin by targeting CDK5. ${ }^{25}$ Furthermore, CDK5 expression was associated with disease prognosis in NPC patients. ${ }^{26}$ These data suggest that CDKAL1 may promote NPC progression by targeting CKD5 in NPC. Further research will be required to validate this hypothesis.

The finding that CDKAL1 expression is a negative predictor of survival in non-metastatic NPC patients should be useful for oncologists in clinical practice. According to the National Comprehensive Cancer Network (NCCN) guidelines (version 1.2020), there are several treatment strategies for non-metastatic NPC including radiotherapy alone, concurrent chemoradiotherapy (CCRT), CCRT combined with neoadjuvant chemotherapy, and CCRT combined with adjuvant chemotherapy. To choose the optimal treatment strategy for each individual patient, additional markers are needed to supplement the TNM staging system. As patients with positive CDKAL1 expression are more likely to have worse OS and PFS, it may be appropriate for these patients to receive more intensive treatments, such as CCRT in combination with neoadjuvant or adjuvant chemotherapy.

It should also be noted that the univariate and multivariate analyses in our study indicated that anti-EGFR targeted therapy and chemotherapy were not independently associated with OS and PFS. With respect to antiEGFR targeted therapy, the survival benefit of adding cetuximab or nimotuzumab to the standard treatment of non-metastatic NPC has garnered considerable controversy, $^{27,28}$ while the $\mathrm{NCCN}$ guidelines (version 1.2020) do not recommend anti-EGFR targeted therapy for non-metastatic NPC due to the lack of high-level evidence. Therefore, more data from well-designed clinical trials are needed to confirm the role of anti-EGFR targeted therapy in non-metastatic NPC. With respect to chemotherapy, there are three options for non-metastatic NPC patients (ie, neoadjuvant, concurrent, and adjuvant chemotherapy). Induction and concurrent chemotherapy have been reported to improve the prognosis of locally advanced NPC in several randomized controlled studies, ${ }^{29,30}$ although the role of adjuvant chemotherapy in non-metastatic NPC is controversial. All of the chemotherapy types were included in the current study, which may explain the non-significant impact of chemotherapy on patient survival.

The results of this study were potentially affected by several factors. First, we did not enroll NPC patients with 
Table 2 Univariate and Multivariate Analyses of Prognostic Factors for OS

\begin{tabular}{|c|c|c|c|c|}
\hline & \multirow[t]{2}{*}{$\mathbf{H R}$} & \multicolumn{2}{|c|}{$95 \% \mathrm{Cl}$} & \multirow[t]{2}{*}{ p-value } \\
\hline & & Lower & Upper & \\
\hline \multicolumn{5}{|l|}{ Univariate analysis $(n=140)$} \\
\hline Age (<45y vs $\geq 45 y)$ & 1.290 & 0.618 & 2.691 & 0.498 \\
\hline Gender (male vs female) & 1.044 & 0.480 & 2.268 & 0.914 \\
\hline CDKALI expression (negative vs positive) & 3.506 & 1.346 & 9.137 & 0.010 \\
\hline Clinical stage & 1.918 & 0.996 & 3.691 & 0.051 \\
\hline Chemotherapy & 1.757 & 0.419 & 7.367 & 0.441 \\
\hline Anti-EGFR targeted therapy & 1.895 & 0.777 & 4.620 & 0.160 \\
\hline Prescription dose of PGTVnx (<70.4 Gy vs $\geq 70.4$ Gy) & 2.980 & 0.406 & 21.885 & 0.283 \\
\hline $\mathrm{T}$ (T3-T4 vs TI-T2) & 1.738 & 0.528 & 5.719 & 0.363 \\
\hline $\mathrm{N}$ (N2-N3 vs N0-NI) & $1.66 \mathrm{I}$ & 0.764 & 3.610 & 0.200 \\
\hline \multicolumn{5}{|l|}{ Multivariate analysis $(n=140)$} \\
\hline CDKALI expression (positive vs negative) & 4.622 & 1.748 & 12.221 & 0.002 \\
\hline Clinical stage & 2.263 & 0.885 & 5.785 & 0.088 \\
\hline Chemotherapy & 1.356 & 0.305 & 6.039 & 0.689 \\
\hline Anti-EGFR targeted therapy & 2.029 & 0.798 & 5.160 & 0.137 \\
\hline T (T3-T4 vs TI-T2) & 0.658 & 0.126 & 3.428 & 0.619 \\
\hline $\mathrm{N}(\mathrm{N} 2-\mathrm{N} 3$ vs $\mathrm{N} 0-\mathrm{NI})$ & 1.282 & 0.572 & 2.877 & 0.546 \\
\hline
\end{tabular}

Abbreviations: OS, overall survival; HR, hazard ratio; $\mathrm{Cl}$, confidence interval; PGTVnx, the planning target volume of the gross tumor volume of nas.

Table 3 Univariate and Multivariate Analyses of Prognostic Factors for PFS

\begin{tabular}{|c|c|c|c|c|}
\hline & \multirow[t]{2}{*}{ HR } & \multicolumn{2}{|c|}{$95 \% \mathrm{Cl}$} & \multirow[t]{2}{*}{ p-value } \\
\hline & & Lower & Upper & \\
\hline \multicolumn{5}{|l|}{ Univariate analysis $(n=140)$} \\
\hline Age (<45y vs. $\geq 45 y)$ & 1.144 & 0.640 & 2.045 & 0.650 \\
\hline Gender (male vs female) & 0.742 & 0.387 & 1.424 & 0.369 \\
\hline CDKALI expression (negative vs positive) & $\mathrm{I} .40 \mathrm{I}$ & 0.771 & 2.546 & 0.269 \\
\hline Clinical stage & 1.758 & 1.077 & 2.869 & 0.024 \\
\hline Chemotherapy & 1.415 & 0.509 & 3.934 & 0.506 \\
\hline Anti-EGFR targeted therapy & 1.805 & 0.876 & 3.722 & 0.109 \\
\hline Prescription dose of PGTVnx (<70.4 Gy vs $\geq 70.4$ Gy) & 2.435 & 0.591 & 10.037 & 0.218 \\
\hline T (T3-T4 vs TI-T2) & 2.276 & 0.818 & 6.331 & 0.115 \\
\hline $\mathrm{N}$ (N2-N3 vs N0-NI) & 1.688 & 0.919 & 3.100 & 0.092 \\
\hline \multicolumn{5}{|l|}{ Multivariate analysis $(n=140)$} \\
\hline CDKALI expression (positive vs negative) & 1.903 & 1.020 & 3.551 & 0.043 \\
\hline Clinical stage & 1.686 & 0.849 & 3.349 & 0.136 \\
\hline Chemotherapy & 1.160 & 0.390 & 3.436 & 0.789 \\
\hline Anti-EGFR targeted therapy & 1.779 & 0.811 & 3.900 & 0.151 \\
\hline $\mathrm{T}$ (T3-T4 vs TI-T2) & 1.098 & 0.285 & 4.241 & 0.892 \\
\hline $\mathrm{N}$ (N2-N3 vs N0-NI) & 1.310 & 0.688 & 2.492 & $0.4 I I$ \\
\hline
\end{tabular}

Abbreviations: PFS, progression-free survival; HR, hazard ratio; $\mathrm{Cl}$, confidence interval; PGTVnx, the planning target volume of the gross tumor volume of nasopharynx.

stage I cancer due to its low incidence. Second, the univariate and multivariate analyses did not include other factors that may affect patient survival, such as the EBVDNA level and expression of Ki-67, due to a lack of available data. Third, the AJCC staging system (7th edition) was adopted in our study, as the 8th edition had not yet been published when the patients were diagnosed. Finally, the sample size was small, so the results may 
have been biased. Further studies including a larger sample of patients will be necessary to confirm our results.

In conclusion, the present study indicated that CDKAL1 expression is an independent negative predictor of survival in non-metastatic NPC patients. Future studies should focus on the specific mechanism underlying the role of CDKAL1 expression in disease outcomes.

\section{Data Sharing Statement}

The datasets used and/or analyzed during the current study are available from the corresponding author on reasonable request.

\section{Ethics Approval and Consent to Participate}

Not applicable.

\section{Patient Consent for Publication}

Not applicable.

\section{Acknowledgments}

We thank LetPub for its linguistic assistance during the preparation of this manuscript.

\section{Funding}

This work was partly supported by the Hunan Department of Science and Technology Foundation, China (YZ: NO. 2018JJ3827). The achievements of this paper are supported by China Scholarship Council (YZ: NO. 201906375015), the Science Foundation of Xiangya Hospital for Young Scholar (ZL: NO. 2018Q012), National Natural Science Foundation of China (ZL: No. 82003239) and (YZ: 82002887), and Hunan Province Natural Science Foundation (Youth Foundation Project) (ZL: NO. 2019JJ50945).

\section{Disclosure}

The authors report no conflicts of interest in this work.

\section{References}

1. Chen YP, Chan A, Le QT, Blanchard P, Sun Y, Ma J. Nasopharyngeal carcinoma. Lancet. 2019;394(10192):64-80. doi:10.1016/S01406736(19)30956-0

2. Wang S, Li S, Shen L. Combined chemoradiation vs radiation therapy alone in stage-II nasopharyngeal carcinoma: a meta-analysis of the published literature. Curr Probl Cancer. 2018;42(3):302-318. doi:10.1016/j.currproblcancer.2018.03.004

3. Bhattacharyya T, Babu G, Kainickal CT. Current role of chemotherapy in nonmetastatic nasopharyngeal cancer. J Oncol. 2018;2018:3725837. doi: $10.1155 / 2018 / 3725837$
4. Jiang Y, Qu S, Pan X, Huang S, Zhu X. Prognostic nomogram for locoregionally advanced nasopharyngeal carcinoma. Sci Rep. 2020;10(1):861. doi:10.1038/s41598-020-57968-x

5. Suzuki C, Blomqvist L, Sundin A, et al. The initial change in tumor size predicts response and survival in patients with metastatic colorectal cancer treated with combination chemotherapy. Ann Oncol. 2012;23(4):948-954. doi:10.1093/annonc/mdr350

6. Zhao Y, Shen L, Huang X, et al. Prognostic analysis of patients with locally advanced nasopharyngeal carcinoma following intensity modulated radiation therapy. Oncol Lett. 2018;15(4):4445-4450. doi:10.3892/ol.2018.7850

7. Locke JM, Wei FY, Tomizawa K, Weedon MN, Harries LW. A cautionary tale: the non-causal association between type 2 diabetes risk SNP, rs7756992, and levels of non-coding RNA, CDKAL1-v1. Diabetologia. 2015;58(4):745-748. doi:10.1007/s00125-015-3508-9

8. Steinthorsdottir V, Thorleifsson G, Reynisdottir I, et al. A variant in CDKAL1 influences insulin response and risk of type 2 diabetes. Nat Genet. 2007;39(6):770-775. doi:10.1038/ng2043

9. Saxena R, Voight BF, Lyssenko V, et al. Genome-wide association analysis identifies loci for type 2 diabetes and triglyceride levels. Science. 2007;316(5829):1331-1336. doi:10.1126/science.1142358

10. Chen MM, O'Mara TA, Thompson DJ, et al. GWAS meta-analysis of 16852 women identifies new susceptibility locus for endometrial cancer. Hum Mol Genet. 2016;25(12):2612-2620.

11. Parada HJ, Cleveland RJ, North KE, et al. Genetic polymorphisms of diabetes-related genes, their interaction with diabetes status, and breast cancer incidence and mortality: the Long Island Breast Cancer Study Project. Mol Carcinog. 2019;58(3):436-446.

12. Lee N, Harris J, Garden AS, et al. Intensity-modulated radiation therapy with or without chemotherapy for nasopharyngeal carcinoma: radiation therapy oncology group Phase II trial 0225. J Clin Oncol. 2009;27(22):3684-3690. doi:10.1200/JCO.2008.19.9109

13. Liu SC, Tsang NM, Chiang WC, et al. Leukemia inhibitory factor promotes nasopharyngeal carcinoma progression and radioresistance. $J$ Clin Invest. 2013;123(12):5269-5283. doi:10.1172/JCI63428

14. Xiao G, Cao Y, Qiu X, Wang W, Wang Y. Influence of gender and age on the survival of patients with nasopharyngeal carcinoma. Bmc Cancer. 2013;13:226. doi:10.1186/1471-2407-13-226

15. Lu X, Wang FL, Guo X, et al. Favorable prognosis of female patients with nasopharyngeal carcinoma. Chin J Cancer. 2013;32(5):283-288. doi:10.5732/cjc.012.10058

16. Dou H, Hu D, Lam C, Liu Y, Wang X, Zhang W. Retrospective analysis of results of treatment for nasopharyngeal carcinoma in Macao. Chin J Cancer Res. 2014;26(2):148-158. doi:10.3978/j. issn.1000-9604.2014.03.01

17. You R, Hua YJ, Liu YP, et al. Concurrent Chemoradiotherapy with or without Anti-EGFR-Targeted Treatment for Stage II-IVb nasopharyngeal carcinoma: retrospective analysis with a large cohort and long follow-up. Theranostics. 2017;7(8):2314-2324. doi:10.7150/thno.19710

18. Lee AW, Law SC, Foo W, Poon YF, Chan DK. Nasopharyngeal carcinoma: local control by megavoltage irradiation. $\mathrm{Br} J$ Radiol. 1993;66(786):528-536. doi:10.1259/0007-1285-66-786-528

19. Sun XS, Liu SL, Luo MJ, et al. The association between the development of radiation therapy, image technology, and chemotherapy, and the survival of patients with nasopharyngeal carcinoma: a Cohort Study From 1990 to 2012. Int J Radiat Oncol Biol Phys. 2019;105 (3):581-590. doi:10.1016/j.ijrobp.2019.06.2549

20. Bellmunt J. Stem-like signature predicting disease progression in early stage bladder cancer. The Role of E2F3 and SOX4. Biomedicines. 2018;6(3):85. doi:10.3390/biomedicines6030085

21. Sainz J, Rudolph A, Hoffmeister M, et al. Effect of type 2 diabetes predisposing genetic variants on colorectal cancer risk. $J$ Clin Endocrinol Metab. 2012;97(5):E845-E851. doi:10.1210/jc.2011-2565

22. Liang Q, Li L, Zhang J, et al. CDK5 is essential for TGF-beta1induced epithelial-mesenchymal transition and breast cancer progression. Sci Rep. 2013;3:2932. doi:10.1038/srep02932 
23. Jia Y, Duan Y, Liu T, et al. LncRNA TTN-AS1 promotes migration, invasion, and epithelial mesenchymal transition of lung adenocarcinoma via sponging miR-142-5p to regulate CDK5. Cell Death Dis. 2019;10(8):573. doi:10.1038/s41419-019-1811-y

24. Sun SS, Zhou X, Huang YY, et al. Targeting STAT3/miR-21 axis inhibits epithelial-mesenchymal transition via regulating CDK5 in head and neck squamous cell carcinoma. Mol Cancer. 2015;14:213. doi:10.1186/s12943-015-0487-x

25. Peng H, Zhang J, Zhang PP, et al. ARNTL hypermethylation promotes tumorigenesis and inhibits cisplatin sensitivity by activating CDK5 transcription in nasopharyngeal carcinoma. J Exp Clin Cancer Res. 2019;38(1):11. doi:10.1186/s13046-018-0997-7

26. Zhang X, Zhong T, Dang Y, Li Z, Li P, Chen G. Aberrant expression of CDK5 infers poor outcomes for nasopharyngeal carcinoma patients. Int J Clin Exp Pathol. 2015;8(7):8066-8074.

27. Li HM, Li P, Qian YJ, et al. A retrospective paired study: efficacy and toxicity of nimotuzumab versus cisplatin concurrent with radiotherapy in nasopharyngeal carcinoma. Bmc Cancer. 2016;16(1):946. doi:10.1186/s12885-016-2974-x
28. Li Y, Chen QY, Tang LQ, et al. Concurrent chemoradiotherapy with or without cetuximab for stage II to IVb nasopharyngeal carcinoma: a case-control study. Bmc Cancer. 2017;17(1):567. doi:10.1186/ s12885-017-3552-6

29. Cao SM, Yang Q, Guo L, et al. Neoadjuvant chemotherapy followed by concurrent chemoradiotherapy versus concurrent chemoradiotherapy alone in locoregionally advanced nasopharyngeal carcinoma: a Phase III multicentre randomised controlled trial. Eur J Cancer. 2017;75:14-23. doi:10.1016/j.ejca.2016.12.039

30. Sun Y, Li WF, Chen NY, et al. Induction chemotherapy plus concurrent chemoradiotherapy versus concurrent chemoradiotherapy alone in locoregionally advanced nasopharyngeal carcinoma: a Phase 3, multicentre, randomised controlled trial. Lancet Oncol. 2016;17(11):1509-1520. doi:10.1016/S1470-2045(16)30410-7

\section{Publish your work in this journal}

Cancer Management and Research is an international, peer-reviewed open access journal focusing on cancer research and the optimal use of preventative and integrated treatment interventions to achieve improved outcomes, enhanced survival and quality of life for the cancer patient.
The manuscript management system is completely online and includes a very quick and fair peer-review system, which is all easy to use. Visit http://www.dovepress.com/testimonials.php to read real quotes from published authors. 